\title{
Organic matter in Seyfert 2 nuclei: Comparison with our Galactic center lines of sight ${ }^{\star}$
}

\author{
E. Dartois ${ }^{1}$, O. Marco ${ }^{2}$, G. M. Muñoz-Caro ${ }^{1}$, K. Brooks ${ }^{3,2}$, D. Deboffle ${ }^{1}$, and L. d'Hendecourt ${ }^{1}$ \\ 1 Institut d'Astrophysique Spatiale, UMR-8617, Université Paris-Sud, Bâtiment 121, 91405 Orsay, France \\ e-mail: emmanuel.dartois@ias.u-psud.fr \\ 2 European Southern Observatory, Alonso de Cordova 3107, Vitacura, Santiago, Chile \\ e-mail: o.marco@eso.org \\ 3 Departamento de Astronomía, Universidad de Chile, Casilla 36-D, Santiago, Chile
}

Received 13 January 2004 / Accepted 19 April 2004

\begin{abstract}
We present ESO - Very Large Telescope and ESA - Infrared Space Observatory 3 to $4 \mu \mathrm{m}$ spectra of Seyfert 2 nuclei as compared to our galactic center lines of sight. The diffuse interstellar medium probed in both environments displays the characteristic $3.4 \mu \mathrm{m}$ aliphatic $\mathrm{CH}$ stretch absorptions of refractory carbonaceous material. The profile of this absorption feature is similar in all sources, indicating the $\mathrm{CH}_{2} / \mathrm{CH}_{3}$ ratios of the carbon chains present in the refractory components of the grains are the same in Seyfert 2 inner regions. At longer wavelengths the circumstellar contamination of most of the galactic lines of sight precludes the identification of other absorption bands arising from the groups constitutive of the aliphatics seen at $3.4 \mu \mathrm{m}$. The clearer continuum produced by the Seyfert 2 nuclei represents promising lines of sight to constrain the existence or absence of strongly infrared active chemical groups such as the carbonyl one, important to understand the role of oxygen insertion in interstellar grains. The Spitzer Space Telescope spectrometer will soon allow one to investigate the importance of aliphatics on a much larger extragalactic sample.
\end{abstract}

Key words. ISM: dust, extinction - ISM: evolution - galaxies: active - galaxies: ISM

\section{Introduction}

Since the first observations (e.g., Allen \& Wickramasinghe 1981) two decades ago, the comparison between infrared features arising from solid state matter, or dust, observed toward many galactic lines of sight, and the studies in the laboratory of materials produced under simulated interstellar conditions has provided some insight into this important component of our Galaxy, which reflects not only its chemical evolution but also radiative transfer effects in many complicated regions. Infrared dust absorption features observed in the Diffuse InterStellar Medium (DISM) and molecular clouds (MC) allow us to infer the solid state matter composition, its evolution, and thus follow the cycling of matter in the Galaxy. Silicates, responsible for the features at $10 \mu \mathrm{m}$ ( $\mathrm{Si}-\mathrm{O}$ stretching mode) and $18 \mu \mathrm{m}$ ( $\mathrm{Si}-\mathrm{O}$ bending mode), as well as carbonaceous material, giving rise to absorption bands around $3.4 \mu \mathrm{m}$ (Pendleton \& Allamandola 2002; Chiar et al. 2000; Pendleton et al. 1994; Sandford et al. 1991) and PAH emission lines (3.3, 6.2, 7.7, 8.6, $11.3 \mu \mathrm{m}$, Leger \& Puget 1984), dominate the composition of refractory dust particles present in the DISM. The relation

* Based on observations collected at the European Southern Observatory, Chile and observations with ISO, an ESA project with instruments funded by ESA Member States. between emission and absorption carbon based observed features has important implications for the cycling of dust in galaxies (Leger et al. 1989).

The exact composition of the carbon-dominated component is still under debate. So far, the identification of the carbonaceous material seen in absorption has proceeded via the spectroscopic analysis of the so-called $3.4 \mu \mathrm{m}$ absorption features due to $-\mathrm{CH}_{2}$ - and $-\mathrm{CH}_{3}$ - aliphatic stretching vibrations in hydrocarbon chains. This $3.4 \mu \mathrm{m}$ absorption is only clearly observed in lines of sight toward our Galactic center (e.g., Chiar et al. 2002, and references therein), or more recently in the foreground diffuse medium toward a luminous Young Stellar Object (YSO) on the shoulder of a strong circumstellar water ice mantle absorption feature (Ishii et al. 2002). In all these lines of sight, it is clear that, in addition to the diffuse interstellar component, high amounts of molecular cloud material and/or circumstellar features also contribute to the extinction, rendering the spectral analysis more complex as one has to decipher the underlying respective contributions of both intervening MC and DISM media. In particular, strong ice mantle absorption features from the densest regions probed by the infrared beam will add several contributions (e.g. $3.1 \mu \mathrm{m}$ water ice, $3.47 \mu \mathrm{m}$ hydrate, $4.27 \mu \mathrm{m} \mathrm{CO}_{2}, 4.67 \mu \mathrm{m} \mathrm{CO}$, $6 \mu \mathrm{m}$ water ice, $6.8 \mu \mathrm{m} \mathrm{CH}_{3}$ deformation modes, methanol, 
$7.7 \mu \mathrm{m}$ methane, $13 \mu \mathrm{m}$ water ice; Dartois et al. 2002; Chiar et al. 2000). To reduce the influence of the MC contribution to the spectra, to perform a finer spectroscopic analysis of the intrinsic DISM absorptions, it is essential to be able to probe DISM on larger galactic scales and not only toward embedded individual infrared sources.

Several observations using the Infared Space Observatory (ISO) spectrometers have allowed the identification of some extragalactic molecular cloud components (Spoon et al. 2002) or extragalactic diffuse components (Laureijs et al. 2000). More recently, ground-based telescopes in specific atmospheric windows clearly demonstrated the extragalactic sources we should focus our attention on. Indeed, the infrared spectra of some external galaxies such as the Seyfert 2 (Sy2) Active Galactic Nuclei show a strong $3.4 \mu \mathrm{m}$ diffuse medium absorption feature, with no major contribution from molecular cloud absorption (e.g. Imanishi 2000).

We present in this paper observations of both galactic and Seyfert 2 extragalactic lines of sight from near $(3.1-4 \mu \mathrm{m})$ to mid (5.4-7.4 $\mu \mathrm{m})$ infrared to investigate the composition of the refractory hydrocarbons present in the interstellar grains. We first present the observations and the observed extragalactic lines of sights in Sects. 2 and 3, then compare in Sect. 4 the observed $3.4 \mu \mathrm{m}$ absorption profiles, in Sect. 5 the carbonaceous to silicates ratios, and the mid-infrared spectra in Sect. 6. We discuss in Sect. 7 the implications for the nature of the DISM hydrocarbons and conclude.

\section{Observations}

The VLT spectra presented here were extracted from the ESO Science Archive Facility (http: //archive.eso.org/) from the programs (69.A-0643 and 69.B-0101) and observations from the authors programs (67.B-0332 and 71.B-0404). A summary of the main parameters are given in Table 1. Data were reduced using in-house software and classical infrared extraction techniques.

The ISO spectra were extracted from the Infrared Space Observatory (ISO) database (http:// isowww.estec.esa.nl/). The different AOT bands were stitched together by applying gain factors, using overlapping regions of the spectra to determine them. The corrections applied were less than 5\%-10\%, in agreement with the differences of the apertures and the absolute photometric reliability of the spectrometer.

\section{Characteristics of the extragalactic sources}

The commonly-accepted AGN model involves a central engine (black hole plus accretion disc) surrounded by sets of dense matter clouds (the so-called broad-line and narrow-line regions, BLR \& NLR) and by a dusty/molecular disc-like and thick structure (also called a torus) which funnels the emission of high energy photons and particles along privileged directions (the ionizing cone, the radio jet axis). This in turn gives rise to viewing-angle dependent effects which are usually invoked to explain the various types of AGN discovered so far (type 1 with broad $\mathrm{H} \alpha$ directly visible or type 2 with broad $\mathrm{H} \alpha$ not directly visible). The spatial extension of the infrared emitting region of Seyfert galaxies is usually in the range 10-100 pc and corresponds to dust heated by the UV emission coming from the accretion disk (sized $\leq 0.01 \mathrm{pc}$ ). The typical extinction on the line of sight is high, $A_{\mathrm{v}}=15-50 \mathrm{mag}$, with a substantial column density of X-ray absorbing material $\left(N_{\mathrm{H}} \sim 10^{22-24} \mathrm{~cm}^{-2}\right)$. ISAAC spectroscopy has been done using a slit width of 1 arcsec, centered on the infrared $L$-band brightest peak of emission of the sources, corresponding to the hot dust $(T \sim 500-1000 \mathrm{~K})$ around the AGN central engine. This means that the slit used with ISAAC is getting all the flux from this region, for all sources.

\section{Individual observed sources}

NGC 1068 is the archetypal Seyfert 2 nucleus, nearby and very bright. It harbours a hidden nucleus with broad permitted emission lines (BLR) seen in spectropolarimetry. The optical-UV luminosity of the central source is $\sim 1.5 \times 10^{45} \mathrm{erg} \mathrm{s}^{-1}$. Adaptive optics observations (Marco \& Alloin 2000) have shown that the hot $(T>900 \mathrm{~K})$ dust emission seems to be confined in a region of $\sim 10 \mathrm{pc}$ radius around the core. $L$-band images show a bright, unresolved central peak of emission surrounded by extended emission, with $90 \%$ of the flux contained in a region less than $100 \mathrm{pc}$ away from the central peak.

NGC 7172 is a Seyfert 2, it has an obscured $\left(N_{\mathrm{H}} \sim 8 \times\right.$ $10^{22} \mathrm{~cm}^{-2}$ ) active nucleus with strong infrared emission from the nucleus $\left(m_{K}=10\right.$ and $\left.L_{\mathrm{IR}} \sim 10^{10.45} L_{\odot}\right)$.

IRAS 19254-7245, also known as The Superantennae, belongs to the Ultra Luminous Infrared Galaxies (ULIRG). Its southern nucleus, the dominant source at different wavelengths, is classified as a Seyfert 2 galaxy, with the presence of a Compton-thick AGN with intrinsic luminosity $10^{44} \mathrm{erg} \mathrm{s}^{-1}$. An image observed with ISOCAM at $6.75 \mu \mathrm{m}$ shows an unresolved central source of an angular size $\leq 3 \mathrm{arcsec}$, with hot dust.

NGC 5506 is Seyfert 2 with hot dust obscuring the central source. A feature of silicate absorption has been detected at $9.7 \mu \mathrm{m}$. The mid-infrared continuum of the nucleus of NGC 5506, is believed to come from warm ( 300 K) dust in thermal equilibrium, which should be located within $\sim 10 \mathrm{pc}$ of the AGN in the case of the UV luminosity with $\sim 10^{11} L_{\odot}$. It has recently been reclassified as an optically-obscured Narrow Line Seyfert 1.

Large scale images of the observed extragalactic objects are presented in Fig. 1 and their main characteristics in Table 2.

\section{3.4 $\mu \mathrm{m}$ absorption feature}

The $3.4 \mu \mathrm{m}$ hydrocarbon absorption features observed in galactic and extragalactic lines of sight are presented in Fig. 2. A local continuum has been subtracted from all spectra, including the wing of a water ice stretching mode absorption band in our Galactic sources lines of sight, as shown by Chiar et al. (2002). All optical depth spectra have been multiplied by a factor, indicated on the left, in order to be able to compare the intrinsic profiles of the bands with an approximately equal optical depth.

The profile observed toward SgR A* with ISO appears peculiar as compared to the others, with more sub-structures. 
Table 1. Source parameters.

\begin{tabular}{|c|c|c|c|c|c|c|c|c|}
\hline Source name & Date & Inst/AOT ${ }^{a}$ & $\mathrm{FOV} /$ slit $^{b}$ & $R$ & $\alpha(2000)$ & $\delta(2000)$ & $z$ & $L \operatorname{Mag}^{c}$ \\
\hline \multicolumn{9}{|l|}{ Galactic center } \\
\hline GC SgrA* & 19 Feb. 1996 & ISO/SWS01 & $14^{\prime \prime} \times 20^{\prime \prime}$ & $R$ & $17 \mathrm{~h} 45 \mathrm{~m} 39.97 \mathrm{~s}$ & $-29 \mathrm{~d} 00^{\prime} 28.7^{\prime \prime}$ & - & 3.1 \\
\hline GC SgrA* & 21 Feb. 1997 & ISO/SWS06 & $14^{\prime \prime} \times 20^{\prime \prime}$ & $R$ & $17 \mathrm{~h} 45 \mathrm{~m} 39.97 \mathrm{~s}$ & $-29 \mathrm{~d} 00^{\prime} 28.8^{\prime \prime}$ & - & \\
\hline GCS 3 I & 29 Aug. 1996 & ISO/SWS01 & $14^{\prime \prime} \times 20^{\prime \prime}$ & $R$ & $17 \mathrm{~h} 46 \mathrm{~m} 14.84 \mathrm{~s}$ & $-28 \mathrm{~d} 49^{\prime} 33.8^{\prime \prime}$ & - & 3.2 \\
\hline GCS 3 I & 08 Oct. 1996 & ISO/SWS06 & $14^{\prime \prime} \times 20^{\prime \prime}$ & $R$ & $17 \mathrm{~h} 46 \mathrm{~m} 14.84 \mathrm{~s}$ & $-28 \mathrm{~d} 49^{\prime} 33.7^{\prime \prime}$ & - & \\
\hline IRAS 17424-2859 & 01 Apr. 1996 & ISO/SWS01 & $14^{\prime \prime} \times 20^{\prime \prime}$ & $R$ & $17 \mathrm{~h} 45 \mathrm{~m} 39.93 \mathrm{~s}$ & $-29 \mathrm{~d} 00^{\prime} 22.8^{\prime \prime}$ & - & 5 \\
\hline GC IRS7 & 29 May 2002 & VLT ISAAC/LWS3 LR & $0.6^{\prime \prime}$ & 600 & $17 \mathrm{~h} 45 \mathrm{~m} 41.1 \mathrm{~s}$ & $-29 \mathrm{~d} 00^{\prime} 44.8^{\prime \prime}$ & - & 5 \\
\hline \multicolumn{9}{|l|}{ Seyfert sources } \\
\hline NGC 1068 & 18 Aug. 2001 & VLT ISAAC/LWS3 LR & $1.0^{\prime \prime}$ & 360 & $02 \mathrm{~h} 42 \mathrm{~m} 40.7 \mathrm{~s}$ & $-00 \mathrm{~d} 00 \mathrm{~m} 48 \mathrm{~s}$ & 0.003793 & 5.4 \\
\hline NGC 7172 & 05 Aug. 2001 & VLT ISAAC/LWS3 LR & $1.0^{\prime \prime}$ & 360 & $22 \mathrm{~h} 02 \mathrm{~m} 01.7 \mathrm{~s}$ & $-31 \mathrm{~d} 52 \mathrm{~m} 18 \mathrm{~s}$ & 0.008683 & 8.5 \\
\hline IRAS 19254-7245 & 04 Jun. 2002 & VLT ISAAC/LWS3 LR & $1.0^{\prime \prime}$ & 360 & $19 \mathrm{~h} 31 \mathrm{~m} 21.4 \mathrm{~s}$ & $-72 \mathrm{~d} 39 \mathrm{~m} 18 \mathrm{~s}$ & 0.061709 & 11.4 \\
\hline NGC 5506 & 18 Jun. 2003 & VLT ISAAC/LWS3 LR & $1.0^{\prime \prime}$ & 360 & $14 \mathrm{~h} 13 \mathrm{~m} 14.87 \mathrm{~s}$ & $-03 \mathrm{~d} 12 \mathrm{~m} 27 \mathrm{~s}$ & 0.006068 & 7.4 \\
\hline
\end{tabular}

${ }^{a}$ AOT: Astronomical Observation Template.

${ }^{b}$ FOV: Field Of View, for ISO-SWS data; slit width for VLT-ISAAC ones.

${ }^{c}$ given $L$ band magnitude are approximate as they are aperture dependent for most of the sources. ISO-SWS spectra are recorded in a source crowded area with a beam size of about $14^{\prime \prime} \times 20^{\prime \prime}$.

This can be explained by the mixing of many infrared emitting sources filling the large observation beam (about 10") of ISO, leading to some confusion in this crowded line of sight (see Eisenhauer et al. 2003, for a recent infrared image of this area), even if there is a dominating infrared source. The two independent GC IRS7 VLT observations, with different orientations of the slit in the sky, better sample the Diffuse Medium by avoiding such a confusion. The $3.4 \mu \mathrm{m}$ profile is therefore better defined.

This profile results from identified absorptions of the symmetric and antisymmetric vibrations of $-\mathrm{CH}_{2-}$ (at $\sim 3.42$ and $3.50 \mu \mathrm{m}$ ) and terminal $-\mathrm{CH}_{3}$ (at $\sim 3.38$ and $3.48 \mu \mathrm{m}$ ) groups in aliphatics, and are indicated above optical depth spectra in Fig. 2. Additional absorption is present around $3.28 \mu \mathrm{m}$, an absorption generally attributed to aromatic $\mathrm{C}-\mathrm{H}$ stretching mode. The resultant optical depth line profiles as seen in NGC 1068, NGC 7172 and NGC 5506 are similar to the ones observed toward GC IRS7 and GCS3I with the VLT, which implies that the $\mathrm{CH}_{2} / \mathrm{CH}_{3}$ aliphatic component ratio of extragalactic dust is the same in the diffuse interstellar medium encountered in these objects, i.e. about $2 \pm 0.5$. In the case of the Superantennae galaxy (IRAS 19254-7245), the $3.4 \mu \mathrm{m}$ is almost saturated $(\tau \sim 0.7)$ but Risaliti et al. (2003) argue that the profile is similar to our Galactic center one.

\section{The $3.4 \mu \mathrm{m}$ hydrocarbons $/ 10 \mu \mathrm{m}$ silicates ratio}

In the analysis of the extragalactic $3.4 \mu \mathrm{m}$, it is common to calculate the ratio of the $3.4 \mu \mathrm{m}$ aliphatic signature of the diffuse galactic medium to the $10 \mu \mathrm{m}$ silicate absorption feature. This ratio should reflect some differences in the carbon to silicate abundances at galactic scales. However, for the ratios to be compared with relevance, one needs an accurate measurement of the scale at which the extinction in both features are measured (e.g., Tomono et al. 2001), as well as to take into account the difference of extension of the infrared background source at 3.4 and $10 \mu \mathrm{m}$. As an example, the comparison of the color excess derived for NGC 7172 by Veilleux et al. (1997) and the silicate absorption observed by the IRAS Low Resolution Spectrometer (Roche et al. 1991) are in contradiction when using classical extinction curves. We used for this particular source a conservative optical depth for the silicates, derived from the color excess, as the silicate optical depth of the IRAS LRS spectrum is poorly constrained. A summary of the $3.4 \mu \mathrm{m} / 9.7 \mu \mathrm{m}$ ratios are given in Table 3 .

Within variations of a factor of two, which could be attributed to galactic plane and/or inner torus dust inclinations as well as temperature gradient effects (which for example modify the optical depth of absorption bands and the scale of the background infrared emitting source at 3 and $10 \mu \mathrm{m}$ ), the $\mathrm{C}_{\text {Aliphatics }} / \mathrm{Si}_{\text {Silicates }}$ ratio in the DISM, as probed by the aliphatics and the silicates, is of the same order.

\section{Galactic center and extragalactic mid infrared spectra}

In a review presented by Pendleton \& Allamandola (2002) are summarised the spectra of most of the interstellar analog relevant material measured in the laboratory, apart from kerogens, and proposed for an interstellar identification of the $3.4 \mu \mathrm{m}$ absorption features. From this set of data, which includes the long wavelength region of these materials, it appears clearly that the simultaneous comparison of the $3-4 \mu \mathrm{m}$ range $\mathrm{C}-\mathrm{H}$ stretching modes absorption with the 5-8 $\mu \mathrm{m}$ bending and deformation modes, "fingerprints" of the same molecules, now acessible via satellite observations, will put the most severe constraint on the 

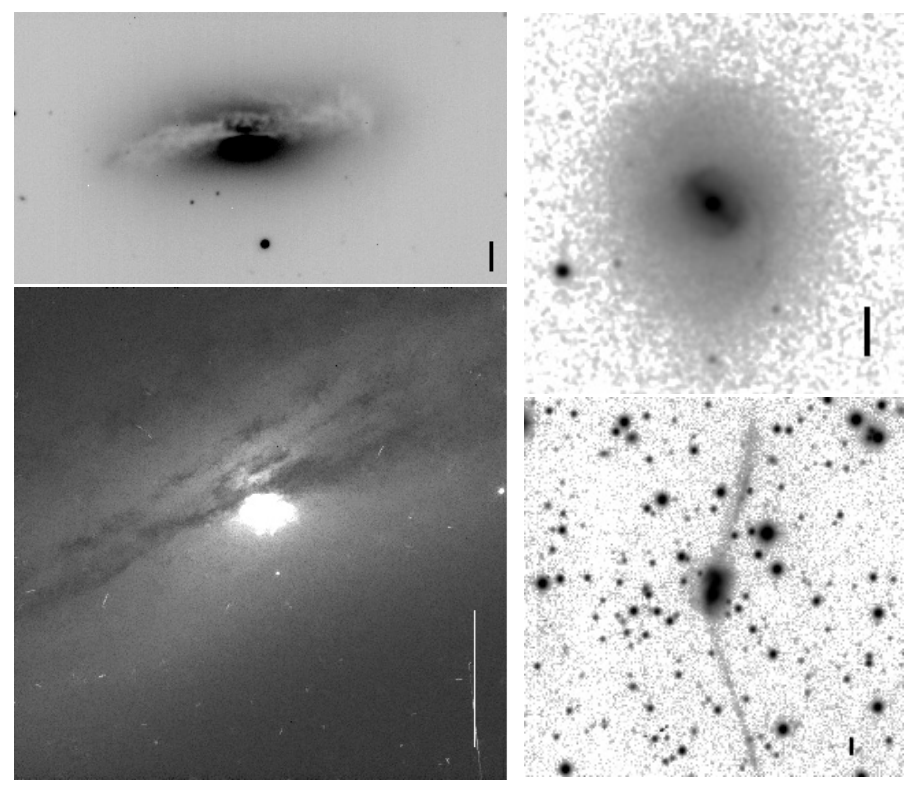

Fig. 1. Images of the observed sources, showing the inclination of the host Seyfert 2 galaxies and where dust obscuration is evident, were retrieved from the NED database (http://nedwww.ipac. caltech.edu/). From left to right, up to down: NGC 7172 (Canada-France-Hawaii Telescope, Hickson (1994)), NGC 1068 (2MASS $K$ band, Jarrett et al. 2003), NGC 5506 (Hubble Space Telescope, $606 \mathrm{~nm}$ filter, Malkan et al. 1998) and IRAS 19254-7245 (Danish Telescope, Chatzichristou 2000). A vertical bar representing a $10^{\prime \prime}$ scale has been drawn.

Table 2. Main characteristics of the extragalactic sources.

\begin{tabular}{lcccc}
\hline \hline Source name & $\begin{array}{c}\text { Distance } \\
\text { Mpc }\end{array}$ & $\begin{array}{c}1^{\prime \prime} \text { equiv. } \\
\mathrm{pc}\end{array}$ & $\begin{array}{c}\text { Size of IR emitting region } \\
\operatorname{arcsec}\end{array}$ & $\begin{array}{c}\text { IR luminosity } \\
\text { Lsol (log 10) }\end{array}$ \\
\hline NGC 1068 & 15 & 73 & $<2.0$ & $11.30^{a}$ \\
NGC 7172 & 35 & 168 & $<2.5$ & $10.45^{b}$ \\
IRAS 19254-7245 & 240 & 1160 & & $12.06^{b}$ \\
NGC 5506 & 24 & 117 & & $10.36^{a}$ \\
\hline
\end{tabular}

${ }^{a}$ Rigopoulou et al. (1999); ${ }^{b}$ Bonatto \& Pastoriza (1997).

nature of the carbonaceous component of the DISM refractive dust. In order to make such a comparison, and to shed light on the line of sight confusion/contamination, in Fig. 3 we show the near and mid-infrared spectra of SgR A*/GC IRS8, GCS3I and NGC 1068, after subtraction of a local continuum due to the background infrared emitting region. The overall optical depth has been normalised to the $3.4 \mu \mathrm{m}$ aliphatic region of the spectrum, in order to directly compare the various absorptions arising in the whole presented spectra.

The mid infrared region of the galactic center individual sources are quite complicated, due mostly to circumstellar and dense molecular cloud materials along the lines of sight that do not participate in the diffuse interstellar medium component. $\mathrm{SgR} \mathrm{A} *$ is dominated in that region by a quite strong water ice bending mode arising in dense molecular cloud environments. This band smears out all the very faint relatively large solid state absorptions that could appear on the continuum of the infrared source. The decomposition of the spectrum with several absorbing components has been reported by Chiar et al. (2000) but clearly the result is dependent on the water ice structure and the number of components used, which are not constrained at all by such a "Gaussian-like" absorption band, making the identification very difficult. The fainter $\mathrm{CH}_{2}$ and $\mathrm{CH}_{3}$ deformation modes are seen at around $6.85 \mu \mathrm{m}$ and $7.25 \mu \mathrm{m}$ and correspond to what is expected for typical aliphatic hydrocarbons. The case of GCS3, presented below and located at only a few arcminutes from $\mathrm{SgR} A *$, is a good example of the problems encountered in the identification of mid infrared spectra. The strong $6.2 \mu \mathrm{m}$ absorption feature observed toward this line of sight is in fact of circumstellar nature, as is observed in several Wolf Rayet envelopes (Chiar \& Tielens 2001), which do not display any strong $3.4 \mu \mathrm{m}$ absorption counterpart.

In contrast to the spectra discussed above, the spectra of the nuclear regions of Seyfert 2 Galaxies do not pertain to the same regime and will not therefore suffer from local circumstellar contamination. The infrared continuum is thought to be emitted by a central dust condensation, of a few parsecs of extension (Tomono et al. 2001; Marco \& Alloin 2000). This offers us the opportunity to probe the matter with a large infrared beam, probing essentially the diffuse matter, and lowering the importance of individual specific objects as they represent a small volume filling factor along the line of sight. The spectrum of the NGC 1068 nucleus, recorded in the mid infrared by the ISO short wavelength spectrometer, and displayed 

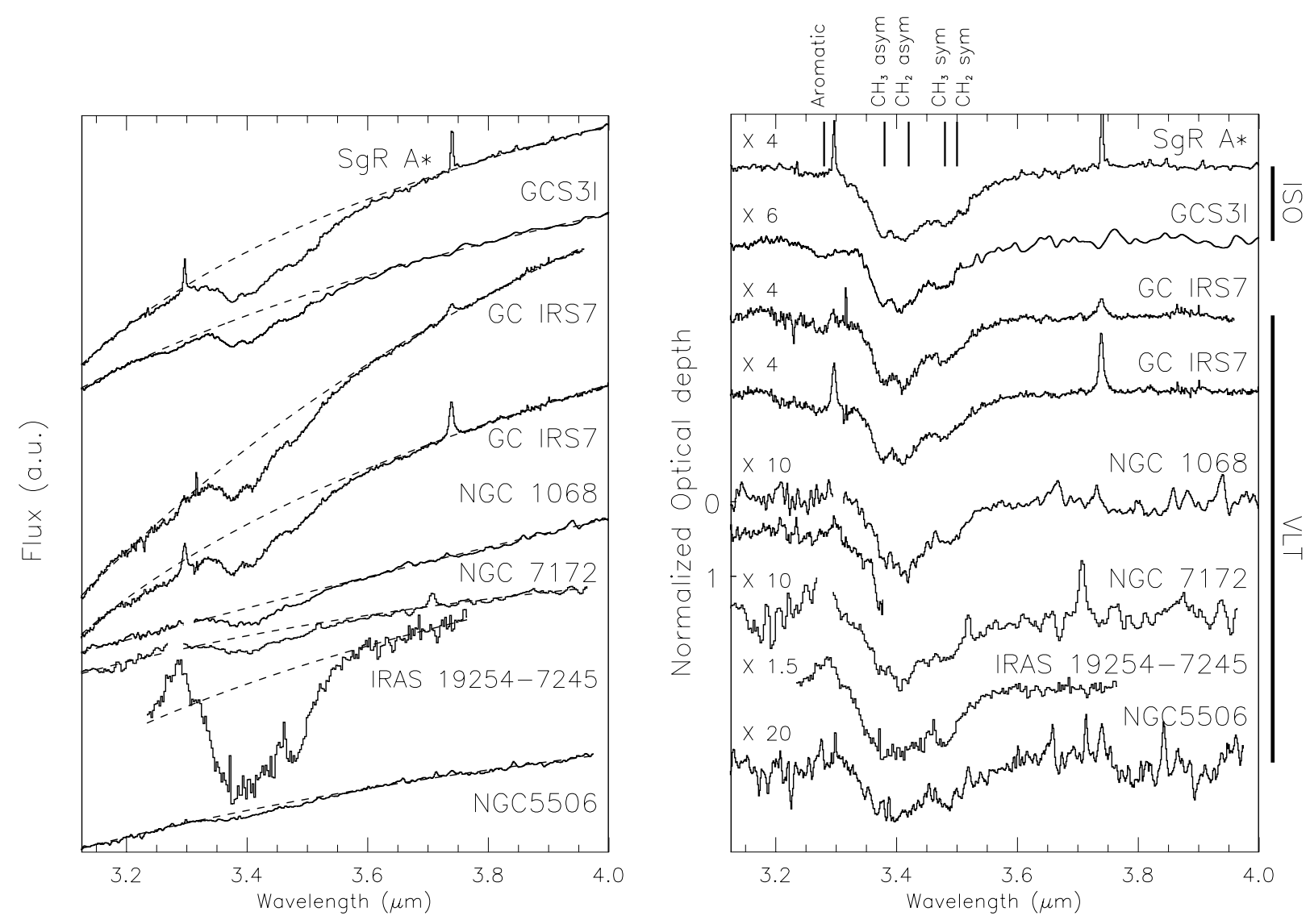

Fig. 2. Left: spectra of the observed sources and adopted local continua to extract the $3.4 \mu \mathrm{m}$ absorption feature, scaled by multiplicative factors for clarity. The $L$ band magnitudes, corresponding to each source, are given in Table 1. The two GC IRS7 independant observations were made with two different slit orientations on the sky and give rise to slightly different continua. Right: normalised optical depth spectra in the 3.15 to $4 \mu \mathrm{m}$ of galactic and extragalactic sources. The two upper traces correspond to ISO Short Wavelength Spectrometer AOT06 spectra $(R \sim 1500)$, except for GCS3I which was combined above $3.5 \mu \mathrm{m}$ with a lower resolution spectrum (SWS01 $R \sim 500$ ). The other spectra are VLT ISAAC Long Wavelength Low resolution spectra $(R \sim 360$ to 600 in the used modes), except the 3.1-3.35 $\mu$ m SWS06 spectrum of NGC 1068 presented below the VLT one. A normalising factor has been applied to allow the comparison of the absorption profiles, and is indicated on the left of each spectra. All extragalactic spectra have been corrected in frequency for their redshift as given in Table 1.

Table 3. Aliphatic hydrocarbons/silicates ratio.

\begin{tabular}{lccc}
\hline \hline $\begin{array}{l}\text { Source } \\
\text { name }\end{array}$ & $\tau(3.4 \mu \mathrm{m})$ & $\tau$ (silicates) & $3.4 \mu \mathrm{m} /$ silicates \\
\hline NGC 1068 & $0.1 \pm 0.015$ & $0.9 \pm 0.1^{a}$ & $0.11 \pm 0.05$ \\
NGC 7172 & $0.09 \pm 0.015$ & $>0.3^{b}$ & $<0.35$ \\
IRAS 19254-7245 & $0.7 \pm 0.1$ & Saturated & - \\
NGC 5506 & $0.04 \pm 0.01$ & $0.9 \pm 0.2^{c}$ & $0.05 \pm 0.2$ \\
\hline
\end{tabular}

${ }^{a}$ Using the value derived by Tomono et al. (2001) in the central $1^{\prime \prime}$ of NGC $1068 ;{ }^{b}$ based on the color excess $E(B-$ $V)$ as explained in Veilleux et al. (1997); ${ }^{c}$ estimated using the Infrared Space Observatory Photometer PHT40 spectrum recorded on 13-Aug.-1996.

in Fig. 3, does not show either the $6 \mu \mathrm{m}$ water ice bending absorption, or the circumstellar contribution at $6.2 \mu \mathrm{m}$ observed for GCS3. The spectrum of the nucleus does present a large absorption around $5.87 \mu \mathrm{m}$ with an optical depth of about half the $3.4 \mu \mathrm{m}$ one. This absorption, intrinsically very strong in the infrared, is typical of the $\mathrm{C}=\mathrm{O}$ carbonyl group and is discussed in relation to the other signatures in the next section.

\section{Constraints on the nature of the hydrocarbons}

\section{The $3.4 \mu \mathrm{m}$ profile}

Given the $\mathrm{CH}_{2} / \mathrm{CH}_{3}$ integrated absorption ratio of about two deduced from the previous analysis, the mean length of the carbon aliphatic chains (sub-chains if branched aliphatics) encountered both in the Galactic and Seyfert 2 galactic nuclei is therefore of six carbon atoms if purely aliphatic, and fewer if attached as individual aliphatic groups to an aromatic structure.

The $3.4 \mu \mathrm{m}$ absorption profile is to a first order compatible with many materials (see for example the Table 3 of Pendleton \& Allamandola 2002). The only firm constraints obtained from these absorption bands are: (i) an estimate of the $\mathrm{CH}_{3}$ to $\mathrm{CH}_{2}$ ratio in the aliphatic component of the ISM dust can be made; (ii) aliphatic chains are interconnected or branched, leading to broad vibrational profiles, as simple aliphatics such as the ones presented in the appendix would display much 

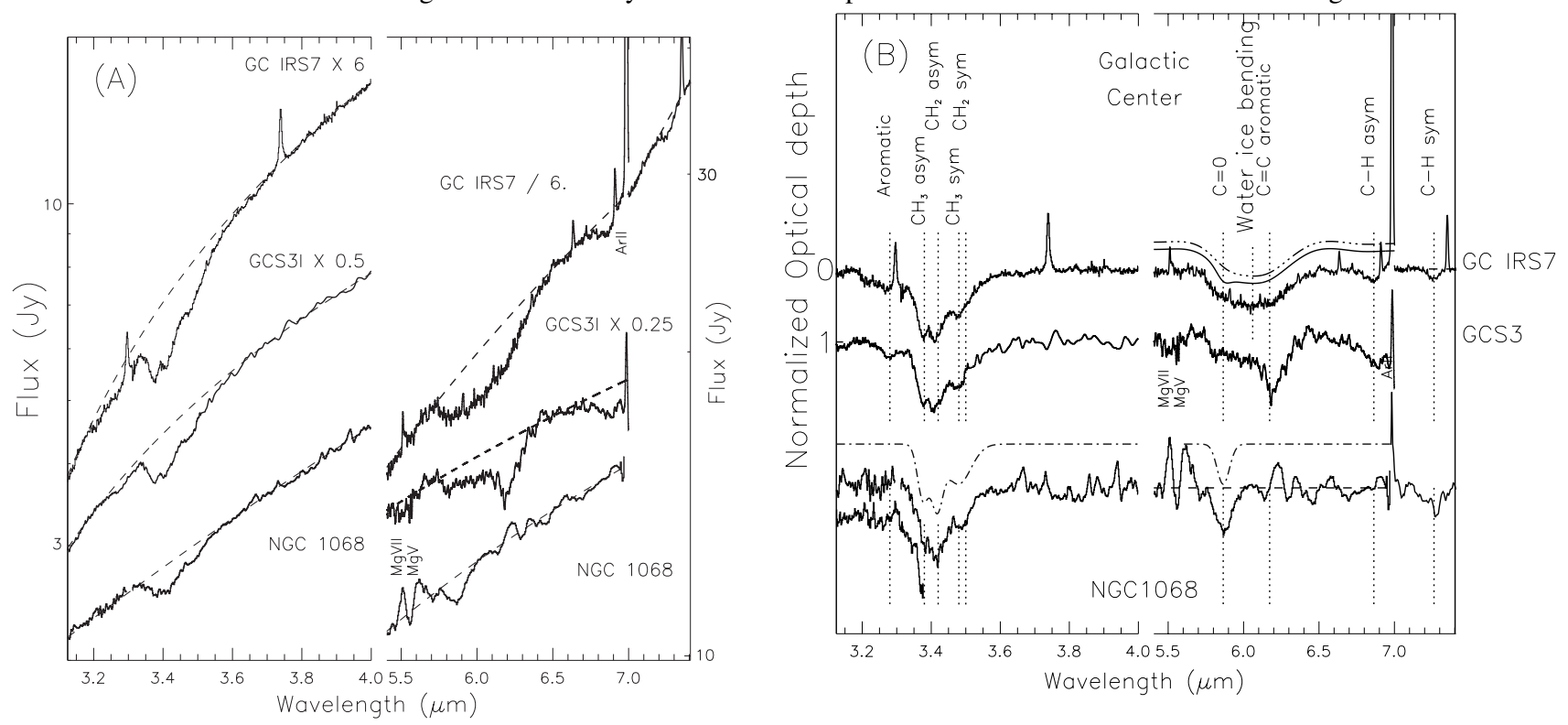

Fig. 3. A): Near and mid infrared spectra of two galactic center lines of sights sampling the diffuse interstellar medium, and one Seyfert 2 inner region. All spectra are scaled as described, for clarity. The adopted local continua used to establish the optical depth in the absorption features are shown as dashed lines. B): Resultant optical depth spectra comparing Galactic center dust absorptions and the one seen along the Seyfert 2 nucleus line of sight of NGC 1068. The NIR (3-4 $\mu \mathrm{m})$ spectra are ISO-SWS or VLT-ISAAC spectra, whereas all MIR (5.5-7.4 $\mu \mathrm{m})$ spectra are ISO-SWS ones. The expected position of caracteristic infrared groups are indicated above. The carbonyl contribution to the IRS7 spectrum which would remain undetected is shown above. It is decomposed in the pure water ice OH bending absorption (dot-dashed) and the maximum undetected carbonyl $+\mathrm{OH}$ (full line). See text for further details.

Table 4. List of detected and/or expected infrared absorption features.

\begin{tabular}{clc}
\hline \hline Wavelength $(\mu \mathrm{m})$ & Mode & Note \\
\hline 3.28 & $\mathrm{C}-\mathrm{H}$ aromatic stretch. & \\
3.38 & $\mathrm{CH}_{3}$ aliphatic asymmetric stretch. & \\
3.42 & $\mathrm{CH}_{2}$ aliphatic asymmetric stretch. & \\
3.48 & $\mathrm{CH}_{3}$ aliphatic symmetric stretch. & \\
3.5 & $\mathrm{CH}_{2}$ aliphatic symmetric stretch. \\
5.87 & $\mathrm{C}=\mathrm{O}$ ketone stretch. \\
6.2 & $\mathrm{C}=\mathrm{C}$ aromatic stretch. \\
6.85 & $\mathrm{CH}_{2}$ aliphatic chain like deform. vibration & \\
7.25 & $\mathrm{CH}_{3}$ aliphatic symmetric bending deform. & \\
9.7 & $\mathrm{SiO}$ silicates stretch. & $c$ \\
\hline
\end{tabular}

${ }^{a}$ Possible contribution from $=\mathrm{CH}$ stretch, mode; ${ }^{b}$ principal more intense component, as less intense $\mathrm{CH}_{3}$ asymmetric deformations occur in this range; ${ }^{c}$ exact position depend on the silicate type.

sharper transitions; and (iii) the aromatic contribution, through its $\mathrm{C}-\mathrm{H}$ stretching component around $3.28 \mu \mathrm{m}$ is at most as abundant as the aliphatic one, except if these aromatics are very large or dehydrogenated.

In addition to the last statement, the $6.2 \mu \mathrm{m}$ aromatic $\mathrm{C}=\mathrm{C}$ vibration is absent or weak from the Galactic center spectra, whereas this mode is much stronger than the aromatic $\mathrm{CH}$ stretch, which implies there is at most a small contribution of aromatics to this DISM material.

\section{Comparison with the carbonaceous phases in meteorites}

The spectra of the insoluble phase of carbonaceous chondrites, such as Orgueil and Murchison, are often considered as excellent candidates analogs for the ISM carbonaceous component, based on a good fit of the $3.4 \mu \mathrm{m}$ of its aliphatic phase (Ehrenfreund et al. 1991; Pendleton 1995). However, the Orgueil and Murchison insoluble material possess a $\mathrm{C} / \mathrm{H}$ ratio of about 17 (Gardinier et al. 2000), which shows that this extracted material is not dominated by aliphatic chains, but rather by aromatic material, as already observed (Gardinier et al. 2000; de Vries et al. 1993). If aliphatic chains were dominant, the $\mathrm{CH}_{2} / \mathrm{CH}_{3}$ network would imply a $\mathrm{C} / \mathrm{H}$ ratio much lower, closer to 0.5 . The long wavelength absorptions in the infrared spectra of these meteorites extracts are a mixture of the aliphatics deformation modes and of the bulk of the aromatics present. When the sample is heated above about $600-700 \mathrm{~K}$ under vacuum, the absorptions seen at $3.4 \mu \mathrm{m}$ disappear whereas most of the strongest absorptions in the 5 to $10 \mu \mathrm{m}$ range remains at higher temperatures (Wdowiak et al. 1988), demonstrating that they are due to at least two different phases, the less volatile being attributed to large polyaromatics. This explains why parts of the Orgeuil and Murchison meteorites are able to reproduce the interstellar $3.4 \mu \mathrm{m}$ profile but fail to account for the now observed longer wavelength part of the spectrum. 


\section{Oxygen content of the DISM aliphatics}

As discussed previously, the NGC 1068 mid infrared spectrum display a carbonyl absorption at $5.87 \mu \mathrm{m}$. The absorption peak position observed is caracteristic of ketones ( $\left.R-(C=O)-R^{\prime}\right)$ or carboxylic acids $(\mathrm{R}-(\mathrm{C}=\mathrm{O})-\mathrm{O}-\mathrm{H})$. The absence of a strong carboxylic $(\mathrm{O}-\mathrm{H})$ feature in the Seyfert 2 spectra, is even more specific to the ketones. Using a decomposition with Gaussians as shown in Fig. 3 of both the 3.4 and $5.87 \mu \mathrm{m}$ integrated absorbance, we can estimate the $\mathrm{C} / \mathrm{O}$ ratio needed to account for this new absorption band. Using $A\left(\mathrm{CH}_{3}\right)=$ $1.25 \times 10^{-17} \mathrm{~cm}$. group ${ }^{-1}$ and $A(\mathrm{C}=0)=2.2 \times 10^{-17} \mathrm{~cm}$ group $^{-1}$ (see Appendix where we determine experimentally the integrated absorbances of ketone carbonyl and $\mathrm{CH}$ stretches; see also d'Hendecourt \& Allamandola 1986) together with the ratio of integrated absorbance deduced from the fit, we obtain a $\mathrm{CH}_{3} / \mathrm{CO}$ ratio of about 3. It leads to a $\mathrm{C} / \mathrm{O}$ of at least 9 taking into account a $\mathrm{CH}_{2} / \mathrm{CH}_{3}$ ratio of about 2 .

The fact we do not see large amounts of oxygen inserted in the aliphatic chains is not so surprising given the oxygen budget in the solid phase of the diffuse medium, where a large part is already locked in the silicates. Indeed, if we use the integrated absorbance values of $A(\mathrm{Si}-\mathrm{O}) \approx 1.6 \times$ $10^{-16} \mathrm{~cm} . \mathrm{molecule}^{-1}$ (Dartois 1998) for the stretching mode of pyroxenes, $\Delta \bar{v} \approx 300 \mathrm{~cm}^{-1}$ for the FWHM of the band, $A_{\mathrm{V}} / \tau_{\text {(silicates } @ 9.7 \mu \mathrm{m})} \sim 16.6$ or 18.5 (Rieke \& Lebofsky 1985; Mathis 1990 ), $N_{\mathrm{H}} / A_{\mathrm{V}} \approx 1.9 \times 10^{21} \mathrm{~cm}^{-2} \mathrm{mag}^{-1}$ (Bohlin et al. 1978), and given that there are 3 oxygen atom per $\mathrm{Si}$ in a pyroxene (the most common pyroxene form is $(\mathrm{Ca}, \mathrm{Mg}, \mathrm{Fe})_{2} \mathrm{Si}_{2} \mathrm{O}_{6}$ ), we can deduce $\mathrm{O} / \mathrm{H} \approx 1.7 \times 10^{-4}$. Using a value of the oxygen atomic abundance of 450 to 550 ppm (Sofia \& Meyer 2001), about $30-40 \%$ of the oxygen is locked in the silicates. A large part of the oxygen seems also confined to the gas phase, in the atomic phase even at moderate to high extinctions (André et al. 2003; Baumgartner 2003).

In infrared spectroscopy, the absence of a line in a spectrum for an infrared active group with a high integrated absorption coefficient (such as the carbonyl) is often a very strong constraint to define the nature of the material, in relation to the detected bands. The appearance of a carbonyl absorption band in the Galactic center lines of sight should not be affected by the water ice observed one, as the former lies in the foreground. This means we can put a useful upper limit on the oxygen content. Based on the mid infrared spectrum of $\mathrm{SgR} \mathrm{A} *$ and using the same carbonyl profile as the one used for NGC 1068, we estimate the maximum $\mathrm{O} / \mathrm{C}$ that would escape detection, as shown in Fig. 3. The upper limit derived in this way translates into an $\mathrm{O}$ (carbonyl $) / \mathrm{C}($ aliphatic $) \leq 40$.

Absence of the ketone carbonyl toward galactic center sources, besides the strength of this infrared absorption, suggests that the synthesis of the carbonaceous ISM is formed in a very reducing environment such as the one shown by Mennella et al. (2002). This is a strong constraint as, for example, almost all the laboratory spectra presented by Pendleton \& Allamandola (2002) present a carbonyl absorption, even the ones in which no oxygen containing molecules were introduced in the experiment, but rather resulted from pollution, showing the ability of oxygen to insert in the aliphatic network and the ease of measuring this particular carbonyl mode.

In summary, observations shown in this paper tend to show that the aliphatic component of Galactic dust and the one encountered toward the nucleus of NGC 1068 are globally the same, except for the possible presence of a small amount of ketones. This last finding should of course be confirmed by further observations at mid infrared wavelength of the Seyfert 2 central dust condensation.

\section{Cycling, timescales and column densities constraints}

Until now, the observations toward the dense medium do not show the presence of any dense cloud $3.4 \mu \mathrm{m}$ absorption, whereas the column densities are large enough to detect it at levels comparable or better than the diffuse medium. Laboratory ice analogues are able to produce refractory materials, stable after the evaporation of the ices, with an efficiency of at most $10^{-2}$ of the initial total ice mass content (e.g., Muñoz Caro \& Schutte 2003, and references therein). Considering an optimistic maximum total ice abundance ratio of a few $10^{-4}$, at most $10^{-6}$ of the total material, with respect to the hydrogen density, is processed in this way in one cycle of a dense cloud. The observed diffuse medium $\mathrm{C}_{\text {aliphatics }} / \mathrm{C}_{\text {total }}$ abundance is around 5 to $10 \%$ (Pendleton et al. 1994; Sandford et al. 1991). One such cycle can therefore produce at most one tenth of the observed DISM aliphatics.

A grain cycling from diffuse to dense medium of at least ten times is required to explain the observations, if we suppose the produced residues are not destroyed and remain in the subsequent cycling sequence. This finding is in contradiction to the fact we do not see the $3.4 \mu \mathrm{m} \mathrm{C}-\mathrm{H}$ absorptions in at least some of the observed dense clouds.

An alternative interesting scenario would be that in the diffuse medium, due to the high atomic hydrogen content in the gas phase, there exists a mechanism that can re-hydrogenate the aliphatic carbon backbone of the otherwise hydrogen UV abstracted material (Mennella et al. 2001, 2002; Muñoz Caro et al. 2001). When entering the dense clouds, the atomic hydrogenation is stopped, due to the passage of a reducing environment dominated by atomic hydrogen to a rather unreactive molecular hydrogen one, and giving rise to a chemistry leading to the formation of the ice mantles. The $\mathrm{CH}$ stretching modes would then disappear. To validate this scenario, one must therefore search for the mid infrared signature, if strong enough, of the much more stable carbon backbone still present, in deeply embedded objects.

\section{Galactic versus extragalactic $3.4 \mu \mathrm{m}$}

In our Galaxy, the $3.4 \mu \mathrm{m}$ absorption feature is sensitive to the environment, as it is not observed in dark clouds. Seyfert 2 and Galactic center lines of sights should probe different mediums, but the solid absorption features in the type 2 Active Galactic Nuclei lines of sight and our Galactic diffuse medium share common properties as underlined by the similarities of their carbonaceous infrared absorption bands. 
AGNs possess a powerful central source (Black hole + accretion disk) that produces the ionizing radiation that heats the dust we see. The temperature of the dust is shown to decrease very fast with the distance to the central source, to reach $100-200 \mathrm{~K}$ at a few hundred parsecs. At the location where the bulk of the absorption takes place, the temperature might not differ too much from the one encountered in the diffuse medium. The material seen via these features must be very UV-resistant and in the solid state as it must stand the strong UV field of the Diffuse Medium. Therefore, only if the temperature of these carbonaceous grains could reach above about $300 \mathrm{~K}$ would we see these lines in emission. The case of the $3.3 \mu \mathrm{m}$, the so called PAH feature, is different as the excitation mechanism is molecular and transient after absorption of single UV photons by relatively small molecular systems.

An important clue toward the understanding of the $3.4 \mu \mathrm{m}$ feature composition lies in the dust cycling timescales of the inner dust torus of Seyfert 2 versus our Galactic diffuse medium. They should be quite different. It seems to favor an interpretation of in-situ formation of this $3.4 \mu \mathrm{m}$ component, without invoking many dust cycles, or the hydrogenation of an almost pure carbon dominated dust, as explained above.

\section{Conclusions}

- The profiles of the aliphatic $3.4 \mu \mathrm{m}$ absorption features measured in four Seyfert 2 nuclei lines of sight are similar to those in our Galaxy, suggesting that both the length of carbon chains and the nature of the $\mathrm{CH}$ stretching modes are similar in these objects.

- The material responsible for our galactic diffuse interstellar medium absorption bands is a relatively simple network containing aliphatics linked together and/or to aromatic structure, and contain little oxygen, as no strong bands arising from other strong infrared active groups are seen in the Mid-Infrared spectra.

- A hint for a carbonyl absorption in the line of sight toward the nucleus of the Seyfert 2 galaxy NGC 1068 is reported. This absorption should be confirmed by further satellite investigations. We recall that use of mid-IR medium resolution spectrometers onboard next generation satellites is the only way to get physical insight into the nature of solid state refractory matter. The almost featureless continuum spectra of some Seyfert 2 nuclei provide excellent lines of sight to search for the mid-IR signatures of the aliphatics seen at $3.4 \mu \mathrm{m}$, an often difficult task for our Galactic center specific lines of sights, due to local circumstellar contamination. Spectra obtained at resolution lower than about 1000 in the mid infrared are of little interest with respect to the exact spectroscopic identification of solid matter composition.

- The numerous extragalactic spectra recorded by the Spitzer Space Telescope will however allow us to obtain the spatial distribution of the DISM solid aliphatic mid-infrared features, and to assess their importance in the cycle of dust at large scales.

Acknowledgements. Part of this work was funded and performed during a visiting scientist program of one month at ESO Chile
Headquarters. The authors wish to thanks the anonymous referee as well as G. Matrajt for fruitful comments that improved the submitted paper.

\section{Appendix: Integrated absorbances of ketones and alcanes at $10 \mathrm{~K}$}

The transmittance $T$ of a plane-parallel film deposited on a non-absorbing thick substrate is a complex function of the wavenumber $\bar{v}$, the substrate refractive index $n_{\mathrm{S}}$, the film refractive index $n_{\mathrm{F}}$, the imaginary part of the film index $k_{\mathrm{F}}$, and the film thickness $d_{\mathrm{F}}$ (Mini 1982). In the regions of the spectrum where absorption is very weak $\left(k_{\mathrm{F}} \ll 1\right)$, this equation takes the simpler form:

$T=\frac{A}{B-C \cos (\phi)+D}$

where $A=16 n_{\mathrm{F}}^{2} n_{\mathrm{S}}, B=\left(n_{\mathrm{F}}+1\right)^{3}\left(n_{\mathrm{F}}+n_{\mathrm{S}}^{2}\right), C=2\left(n_{\mathrm{F}}^{2}-1\right)\left(n_{\mathrm{F}}^{2}-n_{\mathrm{S}}^{2}\right)$, $D=\left(n_{\mathrm{F}}-1\right)^{3}\left(n_{\mathrm{F}}-n_{\mathrm{S}}^{2}\right), \phi=4 \pi n_{\mathrm{F}} \bar{v} d_{\mathrm{F}}$.

This transmittance shows a fringe pattern whose maximum and minimum are given, in the case of $n_{\mathrm{F}}<n_{\mathrm{S}}$, by:

$T_{\mathrm{M}}=\frac{A}{B+C+D}$ and $T_{\mathrm{m}}=\frac{A}{B-C+D}$.

Using the measured transmittance difference between two adjacent maxima, one can therefore obtain the refractive index in a clean $\left(k_{\mathrm{F}} \ll 1\right)$ region of the spectrum by rearranging the above equations in the form:

$n_{\mathrm{F}}=\sqrt{\frac{\gamma \pm \sqrt{\gamma^{2}-4 n_{\mathrm{S}}^{2}}}{2}}$

where $\gamma=\left(1+n_{\mathrm{S}}^{2}+4 n_{\mathrm{S}}\left(1 / T_{\mathrm{M}}-1 / T_{\mathrm{m}}\right)\right)$.

We apply this property of the transmittance to the infrared spectrum of hexane deposited at $10 \mathrm{~K}$ on a CsI substrate, as shown in Fig. 4. The $T_{\mathrm{m}}$ and $T_{\mathrm{M}}$ are evaluated around 1900 and $1000 \mathrm{~cm}^{-1}$, respectively. The CsI substrate refractive index $n_{\mathrm{S}} \sim 1.75$ at cryogenic temperatures. We therefore deduce that $n_{\mathrm{F}} \sim 1.41$ using the above equation.

The thickness can now be evaluated using the wavenumber difference between maxima, as presented in Fig. 4, using the classical interfringe relation $d_{\mathrm{F}}(\mathrm{cm})=1 /\left[2 \Delta \bar{v}\left(\mathrm{~cm}^{-1}\right) n_{\mathrm{F}}\right]$, where $\Delta \bar{v}$ is the wavenumber difference between two adjacent maxima (minima or maxima) of the fringes. The film presented in Fig. 4 has therefore a thickness of $1.86 \mu \mathrm{m}$.

Assuming that $n_{\mathrm{F}}$ does not vary too much in an alkane absorption band, one can now evaluate the integrated absorbance of the infrared active transitions. We subtract a local baseline by modelling the fringes pattern around an absorption and extract the optical depth $\tau$ of the band. The integrated absorbance of a line is now related to this $\tau$ by:

$A(\mathrm{~cm} /$ molecule $)=\int_{\text {line }} \frac{\tau_{\bar{v}} M}{d_{\mathrm{F}} N_{\mathrm{A}} \rho} \mathrm{d} \bar{v}$

and $A(\mathrm{~cm} /$ group $)=\frac{A(\mathrm{~cm} / \text { molecule })}{N_{\mathrm{g}}}$ 

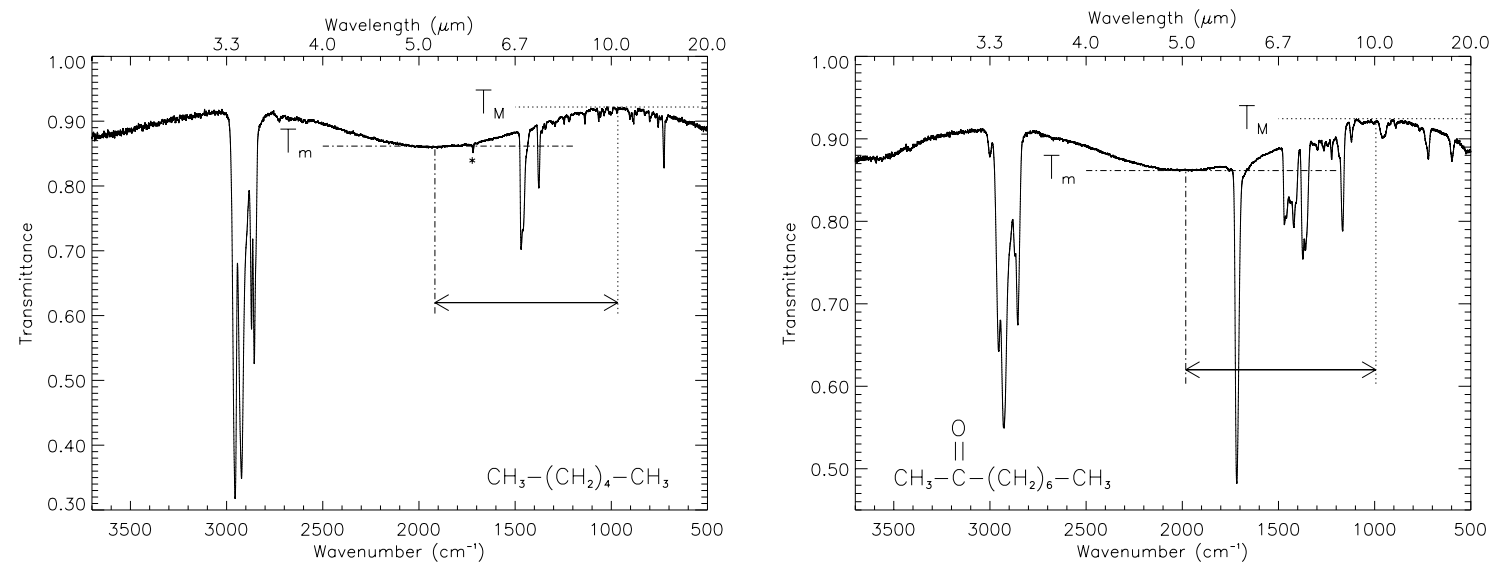

Fig. 4. Transmittance spectra of a film of hexane ice (left) and 2-nonanone ice (right) deposited on a thick CsI window, at $10 \mathrm{~K}$. Note the fringes due to the plane parallel ice film acting as an infrared Fabry-Perot. The meaning and use of the local maxima $T_{\mathrm{M}}$ and $T_{\mathrm{m}}$ as well as their frequency separation indicated on the plot are explained in the text.

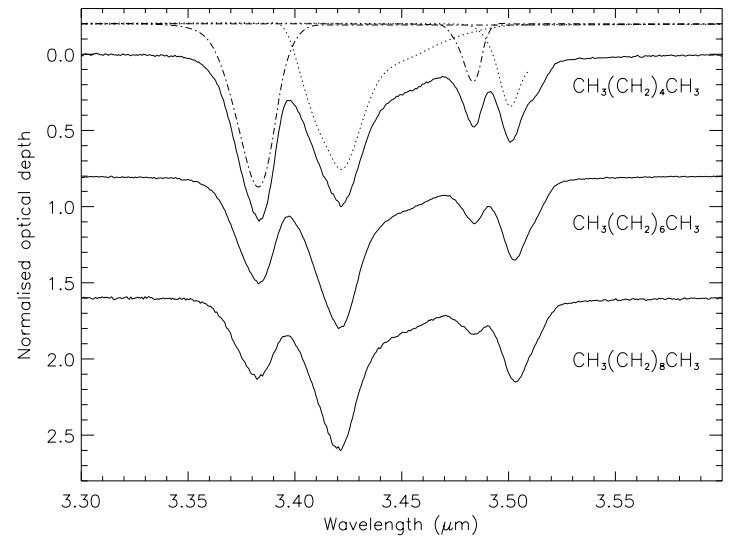

Fig. 5. Optical depth spectra of hexane, octane and decane at $10 \mathrm{~K}$ in the $\mathrm{CH}$ stretch infrared region of the spectrum, after fringes local baseline correction. All spectra have been normalized to the antisymmetrical $\mathrm{CH}_{2}$ stretching mode around $3.42 \mu \mathrm{m}$ for direct comparison of the profiles, and shifted for clarity. The hexane individual stretching modes profiles are displayed in the upper part of the figure. The $\mathrm{CH}_{3}$ and $\mathrm{CH}_{2}$ stretching modes relative ratios are directly proportionnal to the relative contents of the alkanes shown here.

where $M$ is the molar mass, $d_{\mathrm{F}}$ the film thickness, $N_{\mathrm{A}}$ the Avogadro number, $\rho$ the density of the ice and $N_{\mathrm{g}}$ the number of equivalent vibrational groups of the molecule implied in the transition. Using the spectra recorded at our institute at $10 \mathrm{~K}$ of hexane, octane and decane, and after comparison of the baseline subtracted spectra, one can deduce the individual profiles for the various $\mathrm{CH}_{2}$ and $\mathrm{CH}_{3}$ stretching modes, as shown in Fig. 5, where they are normalized to the $\mathrm{CH}_{2}$ antisymmetrical stretch around $2920 \mathrm{~cm}^{-1}$. Using a density of $0.68 \mathrm{~g} \mathrm{~cm}^{-1}$ for the hexane ice, we estimate $A\left(\mathrm{~d}-\mathrm{CH}_{3}\right)=$ $1.25 \pm 0.1 \times 10^{-17} \mathrm{~cm} \mathrm{group}^{-1}$ at $3.38 \mu \mathrm{m}, A\left(\mathrm{a}-\mathrm{CH}_{2}\right)=$ $8.4 \pm 0.1 \times 10^{-18} \mathrm{~cm}$ group $^{-1}$ at $3.42 \mu \mathrm{m}, A\left(\mathrm{~s}-\mathrm{CH}_{3}\right)=2.0 \pm$ $0.1 \times 10^{-18} \mathrm{~cm} \mathrm{group}^{-1}$ at $3.47 \mu \mathrm{m}, A\left(\mathrm{~s}-\mathrm{CH}_{2}\right)=2.4 \pm 0.1 \times$ $10^{-18} \mathrm{~cm}$ group $^{-1}$ at $3.50 \mu \mathrm{m}$.

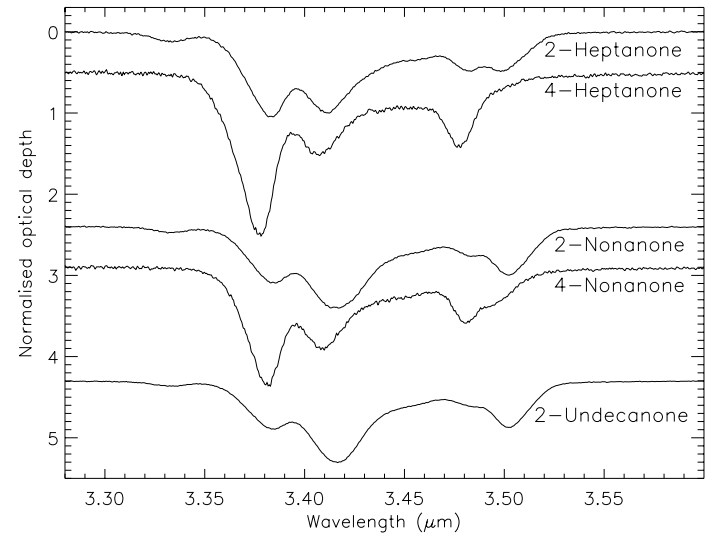

Fig. 6. Optical depth spectra of heptanone, nonanone and undecanone where the carbonyl is either positionned at the end or the middle of the carbon skeleton, after fringes local baseline correction, recorded at $10 \mathrm{~K}$ in the $\mathrm{CH}$ stretch infrared region of the spectrum. All spectra have been normalized to the antisymmetrical $\mathrm{CH}_{2}$ stretching mode around $3.42 \mu \mathrm{m}$ for direct comparison of the profiles, and shifted for clarity.

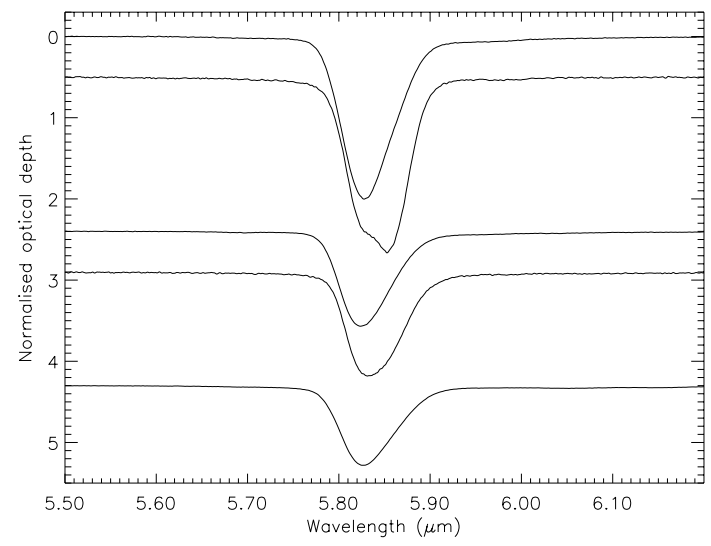

Fig. 7. Optical depth spectra of heptanone, nonanone and undecanone, where the carbonyl is either positionned at the end or the middle of the carbon skeleton, recorded at $10 \mathrm{~K}$ in the $\mathrm{C}=\mathrm{O}$ stretch infrared region of the spectrum. All spectra have been normalized to the antisymmetrical $\mathrm{CH}_{2}$ stretching mode around $3.42 \mu \mathrm{m}$ for direct comparison of the profiles, and shifted for clarity. 


\section{References}

Allen, D. A., \& Wickramasinghe, D. T. 1981, Nature, 294, 239

André, M. K., Oliveira, C. M., Howk, J. C., et al. 2003, ApJ, 591, 1000

Baumgartner, W. H. 2003, Am. Astron. Soc. Meet., 203

Bohlin, R. C., Savage, B. D., \& Drake, J. F. 1978, ApJ, 224, 132

Bonatto, C. J., \& Pastoriza, M. G. 1997, ApJ, 486, 132

Chatzichristou, E. T. 2000, ApJS, 131, 71

Chiar, J. E., Adamson, A. J., Pendleton, Y. J., et al. 2002, ApJ, 570, 198

Chiar, J. E., \& Tielens, A. G. G. M. 2001, ApJ, 550, L207

Chiar, J. E., Tielens, A. G. G. M., Whittet, D. C. B., et al. 2000, ApJ, 537, 749

Dartois, E., d'Hendecourt, L., Thi, W., Pontoppidan, K. M., \& van Dishoeck, E. F. 2002, A\&A, 394, 1057

Dartois, E. 1998, Thesis, Université Paris VI

Ehrenfreund, P., Robert, F., D'Hendencourt, L., \& Behar, F. 1991, A\&A, 252, 712

Eisenhauer, F., Tecza, M., Thatte, N., et al. 2003, The Messenger, 113, 17

Gardinier, A., Derenne, S., Robert, F., et al. 2000, Earth and Planetary Sci. Lett., 184, 9

Dhendecourt, L. B., \& Allamandola, L. J. 1986, A\&AS, 64, 453

Hickson, P. 1994, Atlas of compact groups of galaxies (Gordon and Breach Science Publishers S.A.)

Imanishi, M. 2002, ApJ, 569, 44

Imanishi, M., \& Dudley, C. C. 2000, ApJ, 545, 701

Imanishi, M. 2000, MNRAS, 319, 331

Ishii, M., Nagata, T., Chrysostomou, A., \& Hough, J. H. 2002, AJ, 124,2790

Jarrett, T. H., Chester, T., Cutri, R., Schneider, S. E., \& Huchra, J. P. 2003, AJ, 125, 525

Laureijs, R. J., Watson, D., Metcalfe, L., et al. 2000, A\&A, 359, 900

Leger, A., Dhendencourt, L. B., Verstraete, L., \& Ehrenfreund, P. 1989, Infrared Spectroscopy in Astronomy, 189
Leger, A., \& Puget, J. L. 1984, A\&A, 137, L5

Malkan, M. A., Gorjian, V., \& Tam, R. 1998, ApJS, 117, 25

Marco, O., \& Alloin, D. 2000, A\&A, 353, 465

Mathis, J. S. 1990, ARA\&A, 28, 37

Mennella, V., Brucato, J. R., Colangeli, L., \& Palumbo, P. 2002, Adv. Space Res., 30, 1451

Mennella, V., Muñoz Caro, G. M., Ruiterkamp, R., et al. 2001, A\&A, 367,355

Mini, A. 1982, Thesis, Grenoble

Muñoz Caro, G. M., \& Schutte, W. A. 2003, A\&A, 412, 121

Muñoz Caro, G. M., Ruiterkamp, R., Schutte, W. A., Greenberg, J. M., \& Mennella, V. 2001, A\&A, 367, 347

Pendleton, Y. J., \& Allamandola, L. J. 2002, ApJS, 138, 75

Pendleton, Y. J. 1995, Planet. Space Sci., 43, 1359

Pendleton, Y. J., Sandford, S. A., Allamandola, L. J., Tielens, A. G. G. M., \& Sellgren, K. 1994, ApJ, 437, 683

Rieke, G. H., \& Lebofsky, M. J. 1985, ApJ, 288, 618

Rigopoulou, D., Spoon, H. W. W., Genzel, R., et al. 1999, AJ, 118, 2625

Risaliti, G., Maiolino, R., Marconi, A., et al. 2003, ApJ, 595, L17

Roche, P. F., Aitken, D. K., Smith, C. H., \& Ward, M. J. 1991, MNRAS, 248, 606

Sandford, S. A., Allamandola, L. J., Tielens, A. G. G. M., et al. 1991, ApJ, 371, 607

Sofia, U. J., \& Meyer, D. M. 2001, ApJ, 554, L221

Spoon, H. W. W., Keane, J. V., Tielens, A. G. G. M., et al. 2002, A\&A, 385,1022

Tomono, D., Doi, Y., Usuda, T., \& Nishimura, T. 2001, ApJ, 557, 637

de Vries, M. S., Reihs, K., Wendt, H. R., et al. 1993, Geochim. Cosmochim. Acta, 57, 933

Veilleux, S., Goodrich, R. W., \& Hill, G. J. 1997, ApJ, 477, 631

Wdowiak, T. J., Flickinger, G. C., \& Cronin, J. R. 1988, ApJ, 328, L75 